SU-4240-662

OUTP-98-12P

\title{
Domain Formation in Finite-Time Quenches
}

\author{
Mark Bowick \\ Department of Physics, Syracuse University, \\ Syracuse, NY 13244-1130, U.S.A. \\ Arshad Momen \\ Department of Physics, Theoretical Physics, \\ University of Oxford, 1 Keble Rd., OX1 3NP, U.K.
}

\begin{abstract}
We study the formation of domains in a continuous phase transition with a finite-temperature quench. The model treated is the $\Phi^{4}$ theory in two spatial dimensions with global $O(2)$ symmetry. We investigate this using real-time thermal field theory, following Boyanovsky and collaborators, and find that domain sizes appear to be smaller than those produced in an instantaneous quench in the tree-level approximation. We also propose that a more physical picture emerges by examining the two-point functions which do not involve any cutoff on the short wavelength Goldstone modes.
\end{abstract}

\section{INTRODUCTION}

The formation of domains in phase transitions, and the subsequent (growth) dynamics of these domains is an important topic arising in a variety of contexts ranging from early universe cosmology to condensed-matter physics. As a result, domain formation has been much investigated from both the classical [1] and quantum mechanical [2] points of view.

There are two principal mechanisms of domain formation, namely a) Bubble-Nucleation and b) Spinodal Decomposition. Strongly first order phase transitions typically proceed via bubble-nucleation while spinodal decomposition is often observed in second order phase transitions. For a weakly first order phase transition, however, the idea that the phase transition is driven by the formation of bubbles is not so clear. In fact, this type of phase transition can be driven by spinodal decomposition as well. Nevertheless, domain-type structures appearin both mechanisms.

The growth process of such domains at late times (also known as phase ordering in the literature) is relatively well-understood [1] while the study of the formation process of the defects associated with these domains has begun only recently [3]. The initial size of the 
domains formed in continuous phase transitions is of physical interest. For phase transitions that generate topological defects this domain size also determines the initial defect density.

The earliest attempt to determine the initial domain size, in the cosmological context, is due to Kibble [4,5]. Along with a precise formulation of the mechanism by which topological defects are produced in phase transitions Kibble made a rough estimate, using a thermodynamic equilibrium picture, of typical domain sizes. This was done by determining how big an ordered domain had to be in order to remain stable under thermal fluctuations to the disordered phase. But domain formation is inherently a non-equilibrium process and, therefore, one must be careful about such naive arguments. A more refined viewpoint, based partly on non-equilibrium ideas, has been proposed by Zurek [6]. In the latter picture one focuses on the competition between the applied quench rate and the intrinsic relaxation rate of the order parameter. Defects will freeze out when the order parameter cannot adjust rapidly enough to follow the quench. The correlation length at this freeze-out time determines the characteristic size of ordered domains and hence the typical separation between the resultant topological defects. The non-equilibrium ingredients of this picture imply that the domain size may differ differ significantly from the simple predictions following from thermal stability. Zurek and co-workers have also given numerical evidence for this picture using computer simulations [7].

The processes of domain formation and growth in quantum field theory at finite temperatures have been investigated by Boyanovsky and collaborators [9] using the real-time formalism [10,11]. This formalism is particularly suitable for time-dependent processes like domain formation for which the more popular (imaginary time) Matsubara frequency method is rather cumbersome to use. Applying these techniques for scalar field theories with quartic interactions Boyanovsky et al. [9] managed to reproduce the well-known Cahn-Allen growth laws in the classical theory of phase-ordering [1]. The phase transition in such systems was induced by adopting a sudden quench of the heat bath. Though the quench is performed on the heat-bath rather than on the system, Boyanovsky et al. assumed that the resulting effect was mocked up by taking the mass-function for the scalar fields to be a step-function with a change of sign. Consequently, unstable long-wavelength modes appear in the theory which grow exponentially with time and lead to the appearance of domains.

Scalar field theories admitting global $O(d)$ symmetry admit topological defects in various dimensional spaces. These global topological defects contain singularities of the order parameter which are the zeros of the $O(d)$ symmetric scalar field. The density of such defects is then related to the density of the associated zeros and these can be determined, in a Gaussian approximation, by the Halperin-Mazenko-Liu [12,13 formula, namely

$$
n(t) \sim\left|\frac{W^{\prime \prime}(0, t)}{W(0, t)}\right|^{d / 2},
$$

where $W(x, t)$ is given by the diagonal equal-time two point-function $\left\langle\Phi_{a}(x, t) \Phi_{b}(0, t)\right\rangle \equiv$ $\delta_{a b} W(x, t)$ and the double primes denote the derivative with respect to the radial variable $r \equiv|r|$. Once the two-point function is determined one can use (1.1) to find the defect density [14]. Though one might question the validity of the Gaussian approximation in an interacting system, for $O(n)$ symmetric field theories it can be viewed as a first order result in a more general scheme [15]. 
To be precise, the use of a time-dependent mass-function is flawed as the temperature dependence of the masses is obtained using an equilibrium picture which is definitely not valid with a sudden quench. It is thus interesting to adopt a quench "slow" enough that the approximation to equilibrium remains valid. Though one can expect the late time behavior of the system to be independent of the details of the quench process, this will not be true of the early time behavior of the system. Our goal is to follow the system subsequent to the change in the mass function which we assume to be linear. Such a linear quench has recently been investigated in [16], where it is also found that the defect densities are affected by the existence of a finite time quench - indeed being smaller. Though this result seems correct on physical grounds - its validity can be questioned due to the approximations used. On the other hand, the zeroth order equation of motion can be solved exactly and one can find the two-point function for the unstable modes, following Boyanovsky et. al. [9]. We find that the domain sizes are larger compared to those appearing in a sudden quench, as found by [16] but only during the early part of the quench and towards the end of the quench and thereafter the domains grow more slower in the finite-time quench. This result seems highly counterintuitive as a very slow quench will barely produce defects. On the other hand, the results of [16] show a larger domain size with increasing quench time and hence a lower density of defects.

We argue that this puzzle originates from an inappropriate identification of the Goldstone modes which are associated with the defects - they are not affected by the quench directly. Indeed, we show that by adopting a polar parametrization of the global $O(2)$ model discussed in [16] - the emergent picture is consistent with the scenario proposed by Zurek.

The outline of the paper is as follows. In section II we briefly review the results of Boyanovsky et. al [9], for the case of a sudden quench, to highlight the various approximations used. In section III we generalize to the case of a finite-time quench in their scenario showing that to zeroth order the domain sizes are smaller. In section IV we suggest that more physical results are obtained by studying the correlation functions for the polar fields.

\section{PREDICTIONS FOR A SUDDEN QUENCH}

Let us start from the well-known $S O(2)$ symmetric $\phi^{4}$ theory in $2+1$ dimensions, which admits global vortices since $\pi_{1}\left(S^{1}\right)=Z$. Most of the results below can be found in the paper of Boyanovsky et. al. [9]. The Lagrangian for the system is given by

$$
L=\int d^{3} x\left[\frac{1}{2} \partial_{\mu} \boldsymbol{\phi} \cdot \partial^{\mu} \boldsymbol{\phi}-\frac{1}{2} m^{2}(t) \boldsymbol{\phi} \cdot \boldsymbol{\phi}-\frac{1}{4} \lambda(\boldsymbol{\phi} \cdot \boldsymbol{\phi})^{2}\right]
$$

where $\boldsymbol{\phi} \equiv\left(\begin{array}{l}\phi_{1} \\ \phi_{2}\end{array}\right)$ is a two component vector under $O(2)$ and we have a time-dependent mass function $m(t)$, given by

$$
m^{2}(t)=m_{i}^{2} \Theta(-t)-m_{f}^{2} \Theta(t) .
$$

Due to the $O(2)$ symmetry, the two-point function for this model is diagonal $\left\langle\phi_{a} \phi_{b}\right\rangle=$ $\delta_{a b}\langle\phi \phi\rangle$, where $\langle\phi \phi\rangle$ is the two-point function for a single scalar field. Thus, the results for the $O(2)$ will coincide with those for a single scalar field, treated by Boyanovsky et. al. [9]. 
The zeroth order equation of motion yields

$$
\left[\frac{d^{2}}{d t^{2}}+\mathbf{k}^{2}+m^{2}(t)\right] \mathcal{U}_{k}(t)=0
$$

where $\mathcal{U}_{k}(t)$ is the single particle wavefunction, as defined in the appendix.

With the mass-function defined by (2.2), at times $t<0$, Eqn. (2.3) reads,

$$
\left[\frac{d^{2}}{d t^{2}}+\mathbf{k}^{2}+m_{i}^{2}\right] \mathcal{U}_{k}(t)=0
$$

so that one has as a solution of the form

$$
\mathcal{U}_{k}(t)=e^{-i \omega(k) t},
$$

with $\omega^{2}(k)=\mathbf{k}^{2}+m_{i}^{2}$, which we can treat as an "initial condition".

In the sudden quench scenario, $m^{2}(t)$ changes instantly and at times $t>0$, Eqn. (2.3) reads

$$
\left[\frac{d^{2}}{d t^{2}}+\mathbf{k}^{2}-m_{f}^{2}\right] \mathcal{U}_{k}(t)=0
$$

Clearly, the modes with $\mathbf{k}^{2}<m_{f}^{2}$ are unstable and they are responsible for the formation of domains [17]. For these modes, the solutions to Eqn.(2.6) are given by

$$
\mathcal{U}_{k}(t)=A_{k} e^{W(k) t}+B_{k} e^{-W(k) t},
$$

with

$$
W(k)=\sqrt{m_{f}^{2}-\mathbf{k}^{2}} .
$$

As we are dealing with a second order differential equation, the solutions (2.5) and (2.7) and their first derivatives are required to match at $t=0$, leading to

$$
\begin{aligned}
A_{k} & =\frac{1}{2}\left(1-i \frac{\omega(k)}{W(k)}\right) \\
B_{k} & =\left(A_{k}\right)^{*}
\end{aligned}
$$

where $*$ denotes complex conjugation. For brevity, hereafter we will suppress the functional dependence of $\omega$ and $W$ on $k$.

Given $\mathcal{U}_{k}(t)$, one readily finds for $t>0$, using (A5),

$$
G(\boldsymbol{r}, t)=\frac{1}{2} \int \frac{d^{2} k}{(2 \pi)^{2} 2 \omega} \operatorname{coth}\left(\frac{1}{2} \beta_{i} \omega\right)\left[\left(1+\left(\frac{\omega}{W}\right)^{2}\right) \cosh 2 W t+\left(1-\left(\frac{\omega}{W}\right)^{2}\right)\right] e^{i \boldsymbol{k} \cdot \boldsymbol{r}} .
$$

Note that in the above integral only the unstable modes are taken into account, following [9].

The above integral, however, includes the contributions that are already present before the quench i.e. for $t \leq 0$ and hence is not appropriate for studying the growth of domains. 
One therefore must subtract the contribution that is present at the beginning of the quench. For the unstable mode with wavenumber $k$, the growth is given by the function

$$
\tilde{S}_{k}(t) \equiv\left|\mathcal{U}_{k}(t)\right|^{2}-\left|\mathcal{U}_{k}(0)\right|^{2}
$$

In coordinate space this translates into

$$
\begin{aligned}
\tilde{G}(\boldsymbol{r}, t) & =\int \frac{d^{2} k}{(2 \pi)^{2} 2 \omega} \operatorname{coth}\left(\frac{\beta_{i} \omega}{2}\right) \tilde{S}_{k}(t) e^{i \boldsymbol{k} . \boldsymbol{r}} \\
& =\frac{1}{2} \int \frac{d^{2} k}{(2 \pi)^{2} 2 \omega} \operatorname{coth}\left(\frac{1}{2} \beta_{i} \omega\right)\left[\left(1+\left(\frac{\omega}{W}\right)^{2}\right)(\cosh 2 W t-1)\right] e^{i \boldsymbol{k} . \boldsymbol{r}},
\end{aligned}
$$

which is obtained by replacing $\left|\mathcal{U}_{k}(t)\right|^{2}$ with $\tilde{S}_{k}(t)$ in Eqn. A5).

In the limit of high initial temperatures $(\beta \rightarrow 0)$ and late times, $\tilde{G}(r, t)$ can be evaluated using a saddle point approximation, since the function $k e^{2 W t}$ has a very sharp maximum at

$$
k_{\max } \sim \sqrt{\left(\frac{m_{f}}{2 t}\right)}
$$

This saddle point arises from the competition between the phase space factor $k$ and the growth factor $W(k) t$. Note that this saddle point is absent in one spatial dimension.

An expansion of the integrand around this saddle point allows us to perform the integration at late times, leading to the Gaussian fall-off for the two point function,

$$
\tilde{G}(x, s) \sim \frac{m_{f}\left(1+L^{-2}\right)}{4 \sqrt{2} \pi} J_{0}\left(\frac{x}{\sqrt{2 s}}\right) \frac{e^{2 m_{f} s}}{s} e^{-\frac{x^{2}}{8 s}},
$$

where $L \equiv \frac{m_{i}}{m_{f}}, x \equiv m_{f} r$ and $s \equiv m_{f} t$.

The domain sizes at late times can then be read off by looking at the ratio

$$
D(x, s) \equiv \frac{\tilde{G}(x, s)}{\tilde{G}(0, s)} \simeq e^{-\frac{x^{2}}{8 s}}
$$

where we have used the fact that for $s \rightarrow \infty, J_{0}\left(\frac{x}{\sqrt{2 s}}\right) \rightarrow 1$. Therefore, the domain size at a time $t \gg \frac{1}{m_{f}}$ is given by

$$
\xi(t)=\sqrt{\frac{8 t}{m_{f}}} .
$$

This scaling relation is the same as predicted by the Cahn-Allen equation [1] in the classical theory of domain formation. The result (2.15) requires the existence of a saddlepoint, which is absent in one dimension. It is worthwhile noting that the Cahn-Allen equation also fails in one dimension. In a sense, then, the saddle-point approximation is equivalent to the classical result.

Now as the $\Phi^{4}$ interaction and the associated back reaction effects are ignored, one has to be careful with this zeroth order result. In fact, the zeroth order result has the unphysical feature of indefinite domain growth. This shortcoming, however, cannot be overcome in a 
perturbative framework, as emphasized by Boyanovsky et. al. [9] and others 18. One must treat the problem non-perturbatively using, for example, the Hartree-Fock approximation.

To study the non-perturbative dynamics of domain formation, Boyanovsky et. al. 9] employed a time-dependent Hartree-Fock approximation. Strictly speaking such approximations can only be justified in an $\frac{1}{N}$ expansion for $O(N)$ models. This is definitely questionable for our case with $N=2$. Despite this shortcoming, let us proceed by performing the decomposition

$$
\phi(x, t) \equiv \Phi(t)+\chi(x, t)
$$

in the Lagrangian and retaining only the terms quadratic in the "fluctuation field" $\chi(x, t)$. Note that $\Phi(t)$ gives us the vacuum expectation value of the field $\phi(t)$ at time $t$, i.e.

$$
<\phi(x, t)>=\Phi(t), \quad<\chi(x, t)>=0 .
$$

In the Hartree-Fock approximation one assumes a condensate for the two-point function so that one has

$$
<\phi^{2}(x, t)>\equiv \overline{\Phi^{2}}(t)
$$

The equation of motion for the fluctuation field is now

$$
\left[\frac{d^{2}}{d t^{2}}+\mathbf{k}^{2}+m^{2}(t)+\frac{\lambda}{2} \overline{\Phi^{2}}(t)\right] \mathcal{U}_{k}(t)=0
$$

We also have the condition

$$
\Phi^{2}(t)=\int d^{2} k\left|\mathcal{U}_{k}(t)\right|^{2} \operatorname{coth}\left(\frac{1}{2} \beta \omega\right) .
$$

The set of equations (2.21) and (2.22) have to be solved self-consistently.

Nevertheless, one can study the qualitative behavior of the solution at late times. Note that for a sudden quench, the equation of motion reads, for $t>0$,

$$
\left[\frac{d^{2}}{d t^{2}}+\mathbf{k}^{2}-m_{f}^{2}+\frac{\lambda}{2} \overline{\Phi^{2}}(t)\right] \mathcal{U}_{k}(t)
$$

where we have chosen $\overline{\Phi^{2}}(0)=0$. Since $\overline{\Phi^{2}}$ grows with time as a result of fluctuations, the range of the unstable modes is reduced. At the spinodal time $t=t_{s}$, defined by

$$
m_{f}^{2}=\frac{\lambda}{2} \overline{\Phi^{2}}\left(t_{s}\right)
$$

there are thus no unstable modes in the system and domain growth halts.

\section{INCLUSION OF A FINITE QUENCH}

Realistically any temperature quench occurs within a finite time. How does this affect the above mentioned results? To answer this question, we will assume hereafter that the quench begins at time $t=0$ and ends at time 


\section{FIGURES}

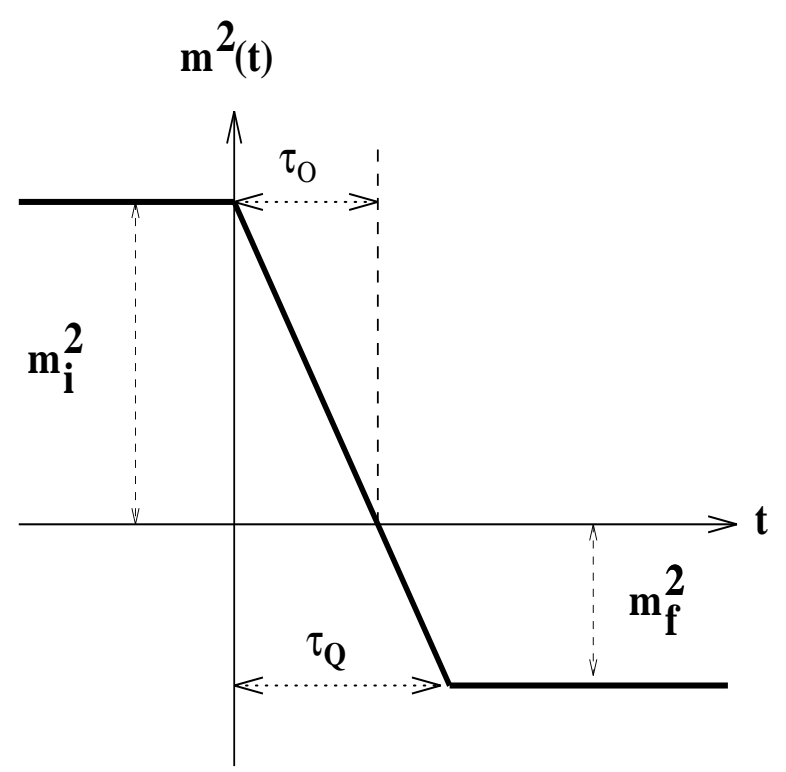

FIG. 1. $m^{2}(t)$ versus time $\mathrm{t}$ for a linear quench

$t=\tau_{Q}$ and it occurs linearly, so that the mass function is of the form

$$
m^{2}(t)=\left\{\begin{array}{l}
m_{i}^{2} \text { for } t \leq 0 \\
m_{i}^{2}-t\left(\frac{m_{i}^{2}+m_{f}^{2}}{\tau_{Q}}\right) \text { for } 0 \leq t \leq \tau_{Q} \\
m_{f}^{2} \text { for } t \geq \tau_{Q}
\end{array}\right.
$$

Let us now define the following quantities:

$$
\begin{aligned}
x & \equiv \frac{\tau_{Q}}{m_{i}^{2}+m_{f}^{2}} \\
y & \equiv x^{\frac{1}{3}} \omega \\
z & \equiv x^{\frac{1}{3}} W,
\end{aligned}
$$

where $\omega$ and $W$ are defined as in (2.5) and (2.8). The solution to the zeroth order equation prior to the quench, for $t \leq 0$, is given by (2.5) as before. During the quench period, $t \in\left[0, \tau_{Q}\right]$, however, the equation of motion at zeroth order reads,

$$
\left[\frac{d^{2}}{d t^{2}}+\mathbf{k}^{2}+m_{i}^{2}-\frac{t}{x}\right] \mathcal{U}_{k}(t)=0
$$

Let us now discuss the consequences of the finite-time quench. 


\section{A. During the quench}

During the quench process, the solution to Eqn. (3.5) can be found in terms of the Airy functions,

$$
\mathcal{U}_{k}(t)=a_{k} A i\left(x^{-\frac{1}{3}}\left(t-\omega^{2} x\right)\right)+b_{k} B i\left(x^{-\frac{1}{3}}\left(t-\omega^{2} x\right)\right) .
$$

The coefficients $a_{k}, b_{k}$ can be determined by matching the solutions (2.5) and (3.6) and their first derivatives at the beginning of the quench, $t=0$ :

$$
\begin{gathered}
a_{k}=\frac{1}{\pi}\left[B i^{\prime}\left(-y^{2}\right)+i y B i\left(-y^{2}\right)\right], \\
b_{k}=-\frac{1}{\pi}\left[A i^{\prime}\left(-y^{2}\right)+i y A i\left(-y^{2}\right)\right],
\end{gathered}
$$

where the prime denotes differentiation with respect to the argument.

The domain growth function can be defined for the finite-time quench similar to (2.11). But, while in the sudden quench the unstable modes appear instantaneously, they will appear with a time lag, in the case of a finite-time quench. This can be understood by noting that in a linear quench the mass function becomes negative at time ( cf. Fig. 1 )

$$
\tau_{0} \equiv \tau_{Q}\left(\frac{m_{i}^{2}}{m_{i}^{2}+m_{f}^{2}}\right)
$$

with the quench beginning at $t=0$. Thus the function that describes the growth of the unstable modes with wave-number $k$ is given by

$$
\tilde{S}_{k}\left(t ; \tau_{0}\right) \equiv\left|\mathcal{U}_{k}(t)\right|^{2}-\left|\mathcal{U}_{k}\left(\tau_{0}\right)\right|^{2}
$$

For an instantaneous quench there is no time lag, i.e. $\tau_{0}=0$, and we get back the standard subtraction [9] in (2.11).

There is another difference between a sudden quench and a finite-time quench. Consider a time $t$, with $\tau_{0}<t<\tau_{Q}$, and mass function $m^{2}(t)=-M^{2}(t)$. The phase space for the

unstable modes is given by $k^{2} \in\left[0, M^{2}(t)\right]$. Note that this interval is smaller than that for the instantaneous quench, as

$$
m_{f}^{2}-M^{2}(t)=\left(m_{i}^{2}+m_{f}^{2}\right)\left[1-\frac{t}{\tau_{Q}}\right]>0 \quad t \in\left[\tau_{0}, \tau_{Q}\right] .
$$

Hence the function describing the domain growth is given by

$$
\bar{G}_{\tau_{0}}(\boldsymbol{r}, t)=\int_{0}^{k^{2}=M^{2}(t)} \frac{d^{2} k}{(2 \pi)^{2} 2 \omega(k)} \operatorname{coth}\left(\frac{\beta_{i} \omega(k)}{2}\right) \tilde{S}_{k}\left(t ; \tau_{0}\right) e^{i \boldsymbol{k} . \boldsymbol{r}},
$$

where the integration is performed over the unstable modes appearing at time $t \in\left[\tau_{0}, \tau_{Q}\right]$.

The domain size can be read off, as before, by looking at the ratio

$$
D_{\tau_{0}}(x, s) \equiv \frac{\bar{G}_{\tau_{0}}(x, s)}{\bar{G}_{\tau_{0}}(0, s)}
$$


In Figs. 2,3,4 a comparative plot of the domain functions for an instantaneous quench and a finite-time quench is given for $\tau_{0}<t<\tau_{Q}$.

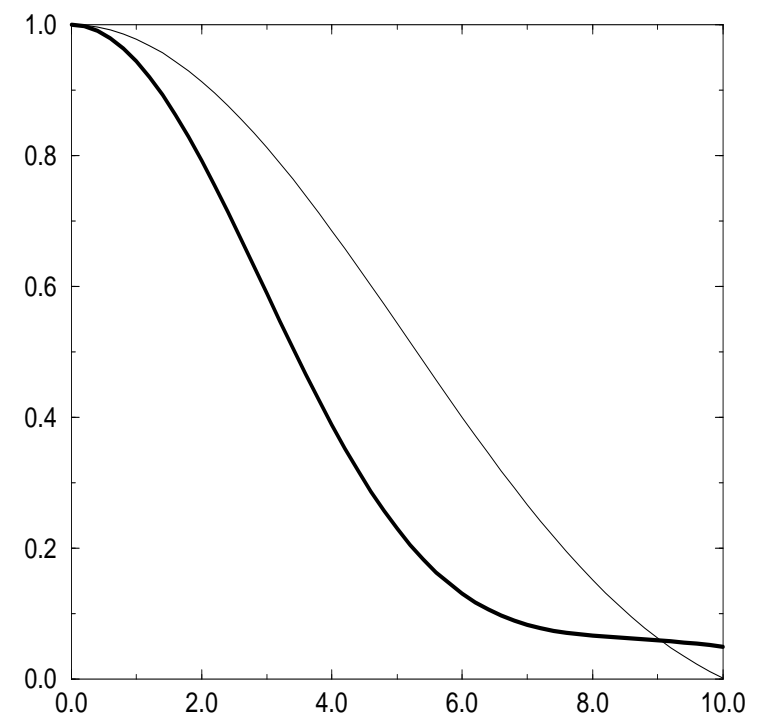

FIG. 2. Comparative plot of $D(x, s)$ and $D_{\tau_{0}}(x, s)$ vs. $x$ with $L \equiv .25$ at $s=.5$ with $s_{Q} \equiv m_{f} \tau_{Q}=1, \beta m_{f}=6$. The thin line corresponds to the case with a finite-time quench while the thick line corresponds to the case with instantaneous quench.

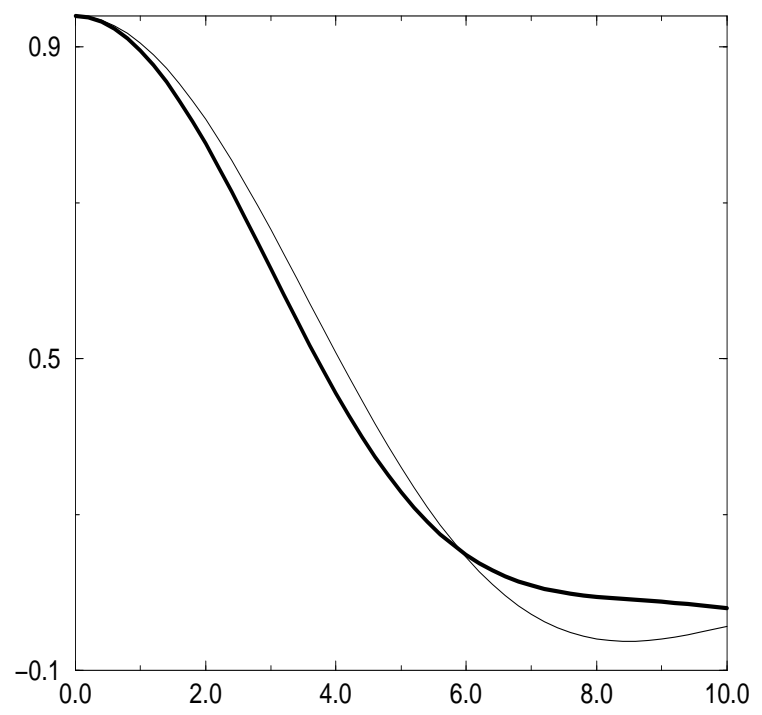

FIG. 3. Same plot as in Fig. 2 but with $\mathrm{s}=.7$ 


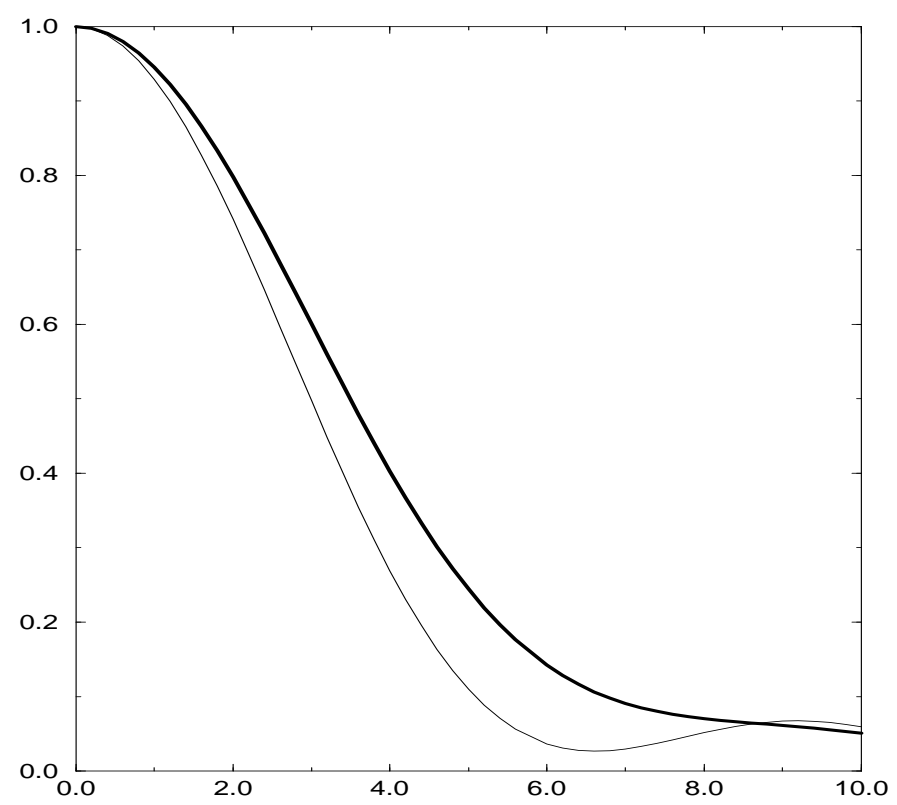

FIG. 4. Same plot as in Fig. 2 but with $\mathrm{s}=.9$

Note that during the early part of the quench, the domain sizes in the finite-quench are larger while later the domain sizes tend to be smaller. This can be explained as a competition between the time lag and the reduced phase space for the unstable modes. Due to the time lag the domains have a shorter time period to grow while due to the smaller phase space the domain function has a larger spread. The latter can be understood by noting that the domain function is defined in position space as a Fourier transform. At early times the phase space is quite small and therefore washes out the effect of the initial time-lag. Domain sizes consequently appear larger than for a sudden quench. On the other hand, at late times, the available phase space for the unstable modes is comparable to that for the sudden quench. At late times the effect of the initial time-lag then becomes clearly visible.

\section{B. After the quench}

At the end of the quench, the equation of motion is given by (2.6), with solutions of the form (2.7).

The coefficients $A_{k}, B_{k}$ can be determined, again, by matching the solutions (3.6) and (2.7) and their first derivatives at the point $t=\tau_{Q}$. This leads to

$$
\begin{aligned}
A_{k} & =\frac{1}{2}\left[a_{k}\left\{A i\left(z^{2}\right)-\frac{1}{z} A i^{\prime}\left(z^{2}\right)\right\}+b_{k}\left\{B i\left(z^{2}\right)-\frac{1}{z} B i^{\prime}\left(z^{2}\right)\right\}\right] e^{\tau_{Q} W}, \\
B_{k} & =\frac{1}{2}\left[a_{k}\left\{A i\left(z^{2}\right)+\frac{1}{z} A i^{\prime}\left(z^{2}\right)\right\}+b_{k}\left\{B i\left(z^{2}\right)+\frac{1}{z} B i^{\prime}\left(z^{2}\right)\right\}\right] e^{-\tau_{Q} W} .
\end{aligned}
$$

The solution after the quench is thus given by

$$
\mathcal{U}_{k}(t)=a_{k}\left[A i\left(z^{2}\right) \cosh W\left(t-\tau_{Q}\right)+\frac{1}{z} A i^{\prime}\left(z^{2}\right) \sinh W\left(t-\tau_{Q}\right)\right]
$$




$$
+b_{k}\left[B i\left(z^{2}\right) \cosh W\left(t-\tau_{Q}\right)+\frac{1}{z} B i^{\prime}\left(z^{2}\right) \sinh W\left(t-\tau_{Q}\right)\right]
$$

where $a_{k}$ and $b_{k}$ are given by (3.7).

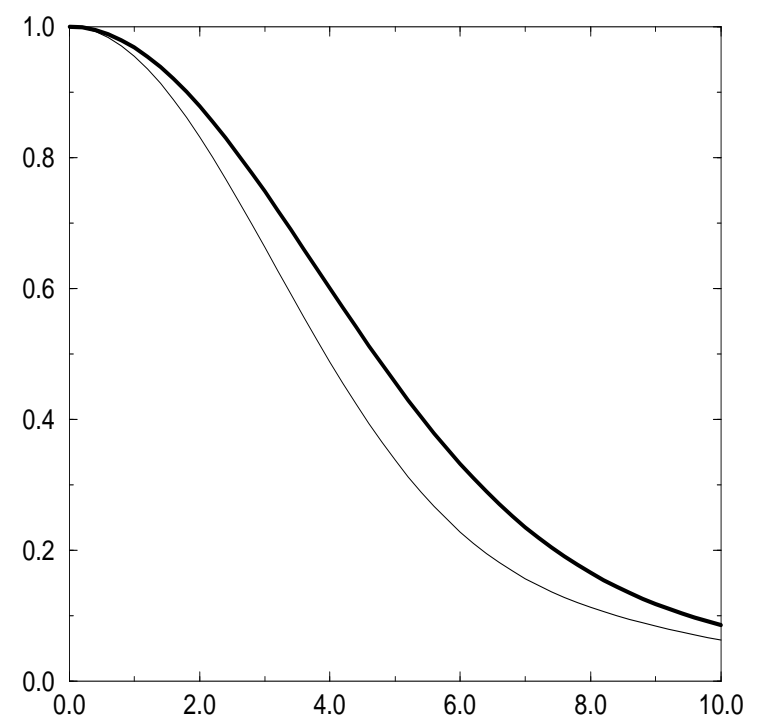

FIG. 5. Same plot as in Fig.2 but with $s=4$

One can again use the domain growth function in (2.11) but with (3.14). As the quench process is finished, however, all the unstable modes with $0<k<M_{f}$ are present after the quench. The domain size is given as before by (3.8). A comparative plot is given in Fig. 5. This shows the domain sizes to be smaller compared with the sudden quench results, as a consequence of their slower growth during the quench period.

This result, however, seems to be in contradiction with the physical picture in which a very slow quench should not lead to appreciable domain formation. This puzzle points out that we have to take into account the effects of the back-reaction.

\section{The role of Back-Reaction}

Let us now incorporate the back-reaction into the picture with a finite-time quench. The equation of motion in the Hartree-Fock theory, during the quench period, would read as

$$
\left[\frac{d^{2}}{d t^{2}}+\mathbf{k}^{2}+m_{i}^{2}+\frac{\lambda}{2} \overline{\Phi^{2}}(t)-\frac{t}{x}\right] \mathcal{U}_{k}(t)
$$

Solving this equation is difficult. To gain some qualitative understanding, let us again consider an adiabatic quench. Note that as $\Phi(t=0)=0$ there is not much change in the initial behavior of system compared to the zeroth order case. As $\Phi(t)$ grows, however, 
one has $\Phi^{2}(t)>0$ and the "back-reaction" competes with the decreasing mass-function, producing a slower quench. Recall that domain formation is associated with the appearance of unstable modes and due to the slower quench they will appear even later compared to the zeroth order case. This is definitely true for small values of $\lambda$, so that the mass function can pass through zero at late times. By our earlier arguments, one can see that the domains formed subsequently will be larger again. On the other hand, for larger values of $\lambda$, if $\Phi$ grows fast, the effective mass-function might not become negative at all during a weak quench and there will be no domain formation. Thus, if defects are to form one would need a quench rate faster than the growth rate for the field $\Phi$, as envisaged in the picture of Zurek [6.

\section{THE QUENCH ON THE GOLDSTONE MODES}

The Halperin-Mazenko-Liu defect density formula involves the complete two-point function whereas we have been dealing with a restricted two-point function which only involves the long-wavelength unstable modes. On the other hand, defects are localized objects and thus they carry short-wavelength modes with them. In fact, the $\mathrm{O}(2)$ global defect is associated with a phase variable which has no apparent cutoff associated with it, as we shall see below.

In our above treatment of the $O(2)$ symmetric scalar model, we have used a Cartesian parametrization for the fields. The zeroth order equations of motion for the two fields accordingly decouple and appear as linear equations. The phase degree of freedom (the Goldstone mode of the theory) nevertheless interacts with the radial mode. The defect configurations in the $O(2)$ model are associated with windings of this Goldstone phase. It can therefore be misleading to use the Cartesian parametrization when studying defect formation. Let us instead rewrite the $\mathrm{O}(2)$ model in terms of a complex field $\Phi$ (polar decomposition):

$$
\Phi \equiv \phi_{1}+i \phi_{2} \equiv F e^{i \Theta}
$$

In terms of $F$ and $\Theta$, the Lagrangian now reads

$$
L=\frac{1}{2}\left[\partial_{\mu} F \partial^{\mu} F+F^{2} \partial_{\mu} \Theta \partial^{\mu} \Theta\right]-\frac{1}{2} m^{2}(t) F^{2}-\frac{1}{4} \lambda F^{4},
$$

exhibiting the Goldstone nature of the phase $\Theta$ and its coupling to the radial mode $F$.

The equations of motion following from the Lagrangian (4.2) are given by:

$$
\begin{aligned}
\partial_{\mu} \partial^{\mu} F+\left[m^{2}(t)+\lambda F^{2}-\left(\partial_{\mu} \Theta \partial^{\mu} \Theta\right)\right] F & =0 \\
\partial_{\mu}\left(F^{2} \partial^{\mu} \Theta\right) & =0
\end{aligned}
$$

Note that in (4.3) the term involving the Goldstone modes appears with the opposite sign to the time-dependent mass function $m^{2}(t)$, in contrast to the case with back-reaction. Note also that the quench does not affect the Goldstone modes directly.

We will attempt to follow the dynamics of this system by using a Born-Oppenheimer like approximation, treating $\Theta$ as a fast variable and $F$ as a slow variable. 
If we ignore the back-reaction due to the field $\Theta$, the $\mathrm{VEV}$ for the radial field $F$ is given at any instant during the quench by

$$
\lambda F^{2}(t)=\left\{\begin{array}{l}
0 \quad(t=0) \\
-m^{2}(t)+\left(\partial_{\mu} \Theta \partial^{\mu} \Theta\right) \quad(t>0)
\end{array}\right.
$$

Using (4.5) in the second equation of (4.4) one gets -

$$
\begin{aligned}
& \lambda \partial_{\mu}\left(F^{2} \partial^{\mu} \Theta\right) \\
& =\partial_{\mu}\left[\left(m^{2}(t)+\left(\partial_{\nu} \Theta \partial^{\nu} \Theta\right)\right) \partial^{\mu}\right] \Theta=0
\end{aligned}
$$

One can attempt to simplify the dynamics by ignoring the $\left(\partial_{\nu} \Theta \partial^{\nu} \Theta\right)$ term in the above equation and solve instead the equation,

$$
\partial_{\mu}\left(m^{2}(t) \partial^{\mu} \Theta\right)=0
$$

where we have dropped the extra term from the equation of motion as it is less relevant at long-wavelength. Equation (4.7) can be expanded as

$$
\ddot{\theta}+\frac{1}{m^{2}}\left(\frac{d}{d t} m^{2}\right) \dot{\theta}-\nabla^{2} \theta=0 .
$$

The second term can be interpreted as a "friction" term which changes sign from positive to negative as the mass function changes sign. Accordingly, there will be production of Goldstone particles which will feed into the dynamics of the $F$ field, as in (4.3). This is similar to the picture in [19]. Since there is no intrinsic mass scale for $\theta$ field one needs to consider all frequencies including the high ones. This is also necessary as the defects are localized objects which can only be probed with high frequencies.

Note that when $m^{2}(t) \rightarrow 0$, only the second term which involves the first derivative in time dominates,

$$
2 \dot{m} \dot{\theta} \approx 0 \quad \text { for } m \rightarrow 0
$$

In the linear quench this shows that the Goldstone modes become time-independent immediately prior to the appearance of the unstable modes. Thus the defects get disentangled from the evolution of the field $F$, as in the picture proposed by Zurek [3].

An adiabatic solution to Eqn. (4.8) is given by

$$
\begin{aligned}
& \theta(x, t)=\int d^{2} k d \omega[\theta(\omega, k)+\text { c.c. }] \\
& \theta(\omega, k)=e^{i \mathbf{k} \cdot \mathbf{x}} e^{-i \omega t}
\end{aligned}
$$

where

$$
\omega \approx \pm\left(k^{2}-\frac{1}{4 m^{4} x^{2}}\right)^{\frac{1}{2}}-\frac{i}{2 m^{2} x}
$$

in the adiabatic approximation, with $x=\frac{\tau_{Q}}{m_{i}^{2}+m_{f}^{2}}$. For the static situation, one has $x=\infty$ and one gets back the standard free-particle. On the other hand, when $m^{2} \rightarrow 0$, we find $\omega \rightarrow 0,-i \infty$ leading to the static situation discussed above. 
Though qualitatively the above scenario supports the picture of defect formation, there is definitely much work needed to get quantitative predictions. Specifically, the domain sizes can be found from the two-point function for the $F$ field. Because of the coupling to the Goldstone modes the evaluation of this two-point function is quite involved. One alternative would be to integrate out the Goldstone field $\Theta$ ( after a Gaussian integration) leading to an effective action involving the $F$ field only

$$
\begin{aligned}
L_{e f f}(F) & =L_{0}-\frac{1}{2} \operatorname{Tr} \ln \left(\partial_{\mu} F^{2} \partial^{\mu}\right) \\
& =L_{0}-\frac{1}{2} \operatorname{Tr} \ln \left(F^{2} \partial^{2}\right)-\frac{1}{2} \operatorname{Tr} \ln \left(1+\frac{\partial_{\mu} F^{2} \partial^{\mu}}{F^{2} \partial^{2}}\right)+\cdots .
\end{aligned}
$$

Note that in the second line we have expanded the logarithm. Note that the operators $F^{2} \partial^{2}$ and $\partial_{\mu} F^{2} \partial^{\mu}$ do not commute and therefore in the above expansion (using the BakerCampbell-Hausdorff formula) there are other terms which are represented by the ellipsis. The second term basically cancels the dependence on $F$ in the functional measure in the path integral. Though naively it seems that the third term in (4.12) suffers from an infrared divergence, the finite temperature of the heat bath provides us with an infrared cutoff. In light of this one can attempt to expand the logarithm in the third term to get an effective action for $F$ which takes into account of the "backreaction" of the Goldstone modes. In practice this is rather complicated as the the functional determinant is time-dependent (due to the time-dependence of the cutoff). For an initial high temperature, the logarithm can

be expanded as $\frac{\partial_{\mu} F \partial^{\mu}}{F^{2} \partial^{2}} \sim \frac{\partial_{\mu} F \partial^{\mu}}{F^{2} T_{i}^{2}}$ is small. During the later stages of the quench, however, this may no longer be true. It might be more appropriate, therefore, to use the recently developed formalism of the non-equilibrium effective action [20] since it generates timedependent correlation functions.

\section{CONCLUSIONS AND REMARKS}

In this letter, we have examined the formation of defects in phase transitions induced by a finite-time quench on $O(2)$ symmetric scalar field theories. We find that adopting the treatment of Boyanovsky et. al. naively with a Cartesian parametrization leads, to the zeroth order, to smaller defect densities during the early quench period while higher defect densities for the later phase. This shows that the problem of the back-reaction needs to be studied properly.

We also propose that a more physical picture will be found by adopting the polar parametrization of the group manifold. This makes explicit the interaction of the true Goldstone phase field with the time-dependent radial field. In the polar parametrization the theory becomes interacting even in the absence of the $\Phi^{4}$ interaction. This makes the analysis of the model much harder. For a finite time quench, however, such a parametrization suggests qualitative support for the Zurek picture of defect formation. This will be valid for the $O(n)$ symmetric scalar models as well. One can attempt to solve the time-dependent model numerically. We hope to report on this in the near future. 


\section{ACKNOWLEDGMENT}

We would like to thank I.J.R. Aitchison, M. Falcioni, A. Kovner, M.C. Marchetti and R.J. Rivers for discussions. A.M. also expresses gratitude to M. Harada for his generous help with MATHEMATICA. This work was supported by the US Department of Energy under contract number DE-FG02-85ER40231 and PPARC (U.K.).

\section{APPENDIX: REAL-TIME FINITE TEMPERATURE FIELD THEORY}

The real time formalism ( also known as the Schwinger-Keldysh formalism) allows one to compute in-in matrix elements, and hence is useful for computing time-dependent averages. For a review of finite temperature field theory in this formalism see, for example, [10]. Here we only quote the formulae needed for our purposes.

In this paper, we will be interested in the equal-time two-point Green function

$$
G(x, t) \equiv<\phi(x, t) \phi(0, t)>=\operatorname{Tr}\left[\rho_{0} \phi(x, t) \phi(0, t)\right]
$$

Here $\rho_{0}$ is the thermal density matrix describing the initial condition,

$$
\rho_{0}=\frac{e^{-\beta H_{i}}}{\operatorname{Tr} e^{-\beta H_{i}}}
$$

with $H_{i}$ the Hamiltonian describing the system initially.

For a bosonic field in $D$ spatial dimensions with a mass $m$ we have the standard expansion:

$$
\phi(x, t)=\int \frac{d^{D} k}{(2 \pi)^{D} 2 \omega(k)}\left[a_{k} \mathcal{U}_{k}(t)+a_{k}^{\dagger} \mathcal{U}_{k}(t)^{\dagger}\right] e^{i \boldsymbol{k} \cdot \boldsymbol{x}}
$$

where $\omega(k)=\left(k^{2}+m^{2}\right)^{\frac{1}{2}}$ and $\mathcal{U}_{k}(t)$ is the one-particle wavefunction in momentum space. The particle creation and destruction operators satisfy

$$
\left[a_{k}, a_{k^{\prime}}^{\dagger}\right]=(2 \pi)^{D} 2 \omega(k) \delta^{D}\left(k-k^{\prime}\right) .
$$

Substituting (A3) in (A1) one gets,

$$
G(x, t)=\int \frac{d^{D} k}{(2 \pi)^{D} 2 \omega(k)} \operatorname{coth}\left(\frac{\beta \omega(k)}{2}\right)\left|\mathcal{U}_{k}(t)\right|^{2} .
$$




\section{REFERENCES}

[1] For a recent review, see A. Bray, Adv. in Phys. 43, 387 (1994) (e-print condmat/9501089).

[2] R. J. Rivers in Formation and Interactions of Topological Defects (Proc. of the NATO Advanced Study Institute on Topological Defects, Newton Institute, Cambridge 1994), eds. A-C. davis and R. Brandenberger, p. 139 (Plenum Press, 1995).

[3] W. Zurek, Phys. Rep. 276, 177 (1996) (e-print cond-mat/9607135).

[4] T. Kibble, J. Physics A 9, 1387 (1977); Phys. Rep. 67, 183 (1980).

[5] M. Hindmarsh and T. Kibble, Rep. Prog. Phys. 58, 477 (1995) (e-print hepph/9411342).

[6] W. Zurek, Nature 317 (1985) 505; Act. Phys. Pol. B 24, 1301 (1993).

[7] P. Laguna and W. Zurek, Phys. Rev. Lett. 78, 2519 (1997) (e-print gr-qc/9607041); Density of Topological defects after a Quench, e-print cond-mat/9705141; Critical Dymanics of Symmetry Breaking: Quenches, Dissipation and Cosmology e-print hep-ph/9711411.

[8] A. Yates and W. Zurek, e-print hep-ph/9801223.

[9] D. Boyanovsky, D.S. Lee and A. Singh, Phys. Rev. D 48, 800 (1993); D. Boyanovsky, Phys. Rev. E 48, 767 (1994).

[10] See, for example, M. Le Bellac, Thermal Field Theory, Cambridge University Press (1996).

[11] E. Calzetta and B.L. Hu, Phys. Rev. D 35, 495 (1987); ibid. D 37, 2878 (1988).

[12] B. I. Halperin, in Physics of Defects, Les Houches 1980, eds. R. Balian, M. Kleman and J.P. Poirer (North-Holland, Amsterdam) 1981.

[13] G. Mazenko and F. Liu, Phys. Rev. B 45, 6989 (1992).

[14] A. J. Gill and R. Rivers, Phys. Rev. D 51, 6949 (1995); T. Evans, A.J. Gill and R. Rivers, e-print hep-ph/9507423.

[15] R. Wickham and G. F. Mazenko, e-print cond-mat/9609163 and references therein.

[16] G. Karra and R. Rivers, Phys. Lett. B 414, 28 (1997) (e-print hep-ph/9705243).

[17] A. Guth and S.Y. Pi, Phys. Rev. D 32, 1899 (1985); E. Weinberg and A. Q. Wu, Phys. Rev. D 36, 2474 (1988).

[18] F. Cooper, S. Habib, Y. Kluger, E. Mottola, J.P. Paz and P. R. Anderson, Phys. Rev. D 50, 2848 (1994) (e-print hep-ph/9405352); F. Cooper, S. Habib, Y. Kluger and E. Mottola, Phys. Rev. D 55, 6471 (1997) (e-print hep-ph/9610345).

[19] D. Boyanovsky, H. J. de Vega, R. Holman, D. S. Lee and A. Singh, Phys. Rev. D 51, 4419 (1995) (e-print hep-ph/9408214); D. Boyanovsky, H. J. de Vega, R. Holman and J. Salgado, Phys. Rev. D 54, 7570 (1996) (e-print hep-ph/9608205).

[20] C. Wetterich, Phys. Rev. Lett. 78, 3598 (1997); Phys. Lett. B 399, 123 (1997); Phys. Rev. E 56, 2687 (1997) (e-print hep-th/9703006); L. Bettencourt and C. Wetterich, e-print hep-ph/9712429. 